\title{
PERSISTÊNCIA DA ATIVIDADE RESIDUAL DO BHC NA SUPERFÍCIE DE DIFERENTES MATERIAIS DE CONSTRUÇÃO
}

\section{Roberto Penna1, Anthony Bosworth ${ }^{1}$, Ibiraci Americano Brasil ${ }^{2}$ e Philip D. Marsden ${ }^{1}$}

\begin{abstract}
Em Mambai (GO), a população faz uso de vários tipos de material de construção. Desde que tem sido detectada uma variabilidade de persistencia da atividade de BHC, foram realizados experimentos laboratorais para testar a capacidade destes materiais em reter a atividade do BHC. Estes experimentos consistiam na aplicação de um kit contendo 10 ninfas de 1. estagio de Dipetalogaster maximus e na verificação do tempo necessário para que houvesse $50 \%$ de mortalidade $\left(T L_{50}\right)$, depois de contatos contínuos com a superficie borrifada. Os testes foram feitos sempre em duplicata, utilizando-se controles adequados. Foram testados um total de 32 superficies em 10 tipos de materiais, com intervalos de tempo até 627 dias após a borrifação.
\end{abstract}

Os resultados obtidos levaram-nos às seguintes conclusōes: 1) a atividade residual do BHC foi detectada, em nosso sistema de teste, até a observação final, 627 dias após a borrifação; 2) esta atividade variava bastante entre os diferentes materiais 88 dias após a borrifação. Esta variação parecia estar relacionada a mudanças na umidade relativa do ar; 3) 'de forma geral, a atividade residual do BHC, em nosso sistema, foi a seguinte, em ordem decrescente: adobe e tijolo não cozido, telha, folhas de palmeira, rebôco sem cal, capim e rebôco com cal, tijolo cozido e madeira.

Palavras chaves: Inseticida. Atividade residual. Triatomineos. BHC.

Nosso interesse neste assunto foi despertado por um pequeno estudo de campo no qual foram utilizados triatomineos sentinelas em contato com superficies borrifadas. para verificar a uniformidade da borrifação e a duração do efeito residual do BHCl1. Técnicas similares têm sido empregadas em outros estudos na literatura 4817 . Em nossa área de estudo uma larga variedade de materiais de construção são usados $910 \mathrm{e}$, desde que tem sido detectada variabilidade na persistência da ação do inseticida 36 , pareceu-nos relevante montar experimentos laboratoriais para estudar este assunto.

\section{MATERIAL E MÉTODOS}

A unidade de teste consistia em uma lata de metal rasa $(0,8 \mathrm{~cm}$ de profundidade $)$ e circular $(5,4 \mathrm{~cm}$ de diâmetro), contendo 10 ninfas de $1 \%$ estágio de Dipetalogaster maximus, envolvida por 2 retalhos de filó (6 espaços/cm). Esta preparação foi aplicada à superficie borrifada continuamente até o fim do experimento. As observações foram feitas diariamente

\footnotetext{
1. Núcleo de Medicina Tropical e Nutrição da Universidade de Brasilia, Brasilia, DF, Brasil.

2. Ministério da Saúde - SUCAM, Formosa, Goiás, Brasil. Trabalho financiado pelo CNPq.

Recebido para publicação em 7/12/1983.
}

por 30 dias e o número de ninfas mortas contado e registrado.

O trabalho inicial de campo foi feito em 10 casas na sede do município de Mambai. Todas as casas tinham sido borrifadas recentemente pela SUCAM. A data exata da borrifação e a quantidade de inseticida usada eram conhecidas e a aplicação foi calculada para que o inseticida fosse distribuído na dose de $500 \mathrm{mg}$ de isômero gama $/ \mathrm{m}^{2}$. Todas as casas eram rebocadas (reboco com cal). Quatro locais para a realização dos testes foram selecionados em cada casa: um na parede da sala, dois nos quartos e mais um na cozinha. As unidades-teste foram mantidas a uma altura de dois metros e examinadas de acordo com o protocolo.

Para examinar a persistência do inseticida em diferentes materiais locais de construção, as seguintes amostras foram coletadas em Mambai: tijolo cozido, adobe, telha, tijolo não cozido, madeira, capim de arroz, dois tipos de palmeira (Acrocomia sclerocarpa e Mauritia vinifera) e rebôco com cal e sem cal. Estes materiais foram trazidos para o laboratório onde foram mantidos sob condições ambientais e protegidos da luz. Dados de temperatura e umidade foram obtidos no Instituto Nacional de Meteorologia (INEMET). Os materiais foram arranjados de forma à constituírem 
Penna R, Bosworth A, Brasil IA, Marsden PD. Persistência da atividade residual do BHC na superficie de diferentes materiais de construção. Revista da Sociedade Brasileira de Medicina Tropical 17: 95-99, Abr-Jun, 1984

superficies uniformes, mais adequados para os testes e foram borrifadas segundo o protocolo da SUCAM. As mesmas áreas de teste foram usadas em todos os experimentos e cada unidade foi aplicada em duplicata sobre cada material e observada diariamente por 30 dias. Duas unidades de controle, aplicadas à superficies não borrifadas, foram observadas de maneira semelhante. Dois lotes de amostras de materiais foram testados. Um foi borrifado em maio de 1981, no inicio da seca, enquanto que o outro foi aspergido em outubro de 1981, no inicio das chuvas. Os experimentos foram realizados até 627 dias após a borrifação. Os resultados foram expressos em termos do número de dias necessários para que houvesse $50 \%$ de mortalidade (TL50).

\section{RESULTADOS}

A Tabela 1 mostra dados de TL50 de 10 casas no primeiro procedimento de campo. Uma semana após a borrifação todos os barbeiros morreram nas primeiras 24 horas, com exceção da caixa colocada na sala da casa de número 5 . Ao que parece este foi o único lugar, dos 40 estudados, que foi borrifado de forma inadequada. Nos meses seguintes a atividade do inseticida declinou, mas persistiu em niveis detectáveis até 7 meses depois da borrifação. Uma variação no poder tóxico ocorreu durante o periodo de teste e foi verificada pelo TL50 médio dos testes do $3{ }^{\circ}$ ao 7 ? mês.

As Tabelas 2 e 3 apresentam dados relacionados com o TL50 de diferentes superficies borrifadas nas duas ocasiões e observadas no laboratório sequencialmente em intervalos até 627 dias. 0 mesmo declínio na toxicidade com o tempo foi observado, mas a variação dos resultados é mais clara na Tabela 2 . Após 390 dias da borrifação, apenas dois materiais registraram TL50 em 30 dias: adōbe e buriti. Entretanto, 568 dias após a borrifação, todos os materiais voltaram a registrar o TL50, sendo esta época o periodo de chuvas.

A Figura 1 ilustra os resultados da Tabela 3, que mostra a atividade do BHC em determinadas amostras de materiais relacionada com as condições ambientais. Uma curva pode ser claramente observada e mostra variações no TL50 refletindo a variação da atividade do inseticida. Assim, a 240 e 340 dias após a borrifação, exatamente naqueles periodos nos quais a pluviosidade foi menor, 0 e $80,4 \mathrm{~mm}^{3}$ respectivamente, as amostras levaram mais tempo para atingir o TL50.

Tabela 1 - Dados de $\mathrm{TL}_{50}{ }^{+}$em dias, de 10 casas borrifadas com BHC em Mambaí (GO). Data da borrifação: 19/8/1980.

\begin{tabular}{|c|c|c|c|c|c|c|c|c|}
\hline \multirow{2}{*}{ Casas } & \multicolumn{8}{|c|}{ Tempo após a borrifação } \\
\hline & 1 semana & 1 mês & 2 meses & 3 meses & 4 meses & 5 meses & 6 meses & 7 meses \\
\hline 1 & 1 & 1 & 3 & 2,2 & 2,2 & 1 & 7,2 & 4,2 \\
\hline 2 & 1 & 1 & ++ & 2,2 & 4 & ++ & ++ & ND \\
\hline 3 & 1 & 1 & 1,7 & 2,2 & 1,5 & 1 & 3,5 & 3,5 \\
\hline 4 & 1 & 1 & 2,7 & 2 & 1,5 & 1,7 & 6 & 5 \\
\hline 5 & 1,5 & 1,2 & 1 & 2,2 & 3,2 & 1,2 & 5,2 & ND \\
\hline 6 & 1 & 1 & 1,7 & 2,2 & 2,5 & 2,7 & 8,7 & ND \\
\hline 7 & 1 & 1 & 3,2 & 2,7 & 5,5 & 4,7 & 9 & 14,5 \\
\hline 8 & 1 & 1 & 1,2 & 1,5 & 3,7 & 2,7 & 4,5 & 5,2 \\
\hline 9 & 1 & 1 & 1,2 & 1,7 & 1,5 & 1 & 3 & 4 \\
\hline 10 & 1 & 1 & 1,2 & 2 & 2 & 3 & 6 & ND \\
\hline $\mathrm{TL}_{50}$ & & & & & & & & \\
\hline Média & 1 & 1 & 1,9 & 2,1 & 2,8 & 2,1 & 5,9 & 6,1 \\
\hline
\end{tabular}

$+\mathrm{TL}_{50}$ - Tempo necessário para que haja $50 \%$ de mortalidade.

++- Casa fechada.

ND - Não há dados. 
Penna R, Bosworth A, Brasil IA, Marsden PD. Persistencia da atividade residual do BHC na superficie de diferentes materiais de construção. Revista da Sociedade Brasileira de Medicina Tropical 17: 95-99, Abr-Jun, 1984

Tabela 2 - Dados de $T L_{50}{ }^{+}$e dados de temperatura média, umidade relativa do ar e pluviosidade no Distrito Federal ++. Material borrifado em 19/5/1981.

\begin{tabular}{|c|c|c|c|c|c|c|c|c|c|}
\hline \multirow{2}{*}{$\begin{array}{l}\text { Material e dados } \\
\text { meteorológicos }\end{array}$} & \multicolumn{9}{|c|}{ Dias após a borrifação } \\
\hline & 7 & 30 & 88 & 152 & 283 & 390 & 490 & 568 & 627 \\
\hline Capim de arroz & 1 & 1 & 1 & 3 & 5,5 & +++ & +++ & 23,5 & 13,5 \\
\hline Acrocomia sclerocarpa & 1 & 1,5 & 1,5 & 2,5 & 7,5 & +++ & 21 & 23 & 13 \\
\hline Tijolo cozido & 1 & 1 & 15,5 & 7 & 17 & +++ & +++ & 22 & 18,5 \\
\hline Adobe & 1 & 1 & 1,5 & 2 & 3,5 & 24 & 20 & 11,5 & 5 \\
\hline Telha & 1 & 1 & 4 & 4 & 11,5 & $++t$ & +++ & 27 & 22 \\
\hline Madeira & 1 & 1 & 4 & 4 & 10 & $+t+$ & +++ & 17 & 11,5 \\
\hline Mauritia vinifera & 1 & 1 & 1,5 & 3 & 9 & 28,5 & 19 & 20,4 & 12 \\
\hline Mortalidade controle & +++ & +++ & +++ & +++ & +++ & +++ & +++ & +++ & +++ \\
\hline \multicolumn{10}{|l|}{ Temperatura } \\
\hline média $\left({ }^{\circ} \mathrm{C}\right)$ & 18,9 & 17,8 & 19,7 & $\begin{array}{l}20,5 \\
58\end{array}$ & $\begin{array}{l}21,1 \\
81\end{array}$ & 19,4 & ND & $\begin{array}{l}20,9 \\
N D\end{array}$ & $\begin{array}{l}21,4 \\
84\end{array}$ \\
\hline $\begin{array}{l}\text { Umidade relativa } \\
\text { Pluviosidade }\left(\mathrm{mm}^{3}\right)\end{array}$ & $\begin{array}{l}70 \\
17.8\end{array}$ & $\begin{array}{l}48 \\
22,4\end{array}$ & $\begin{array}{l}49,3 \\
1,3\end{array}$ & $\begin{array}{l}58 \\
437.5\end{array}$ & $\begin{array}{l}81 \\
233,4\end{array}$ & $\begin{array}{l}62 \\
0\end{array}$ & $\begin{array}{l}\text { ND } \\
80.4\end{array}$ & $\begin{array}{l}\text { ND } \\
160.2\end{array}$ & $\begin{array}{l}84 \\
207.3\end{array}$ \\
\hline Pluviosidade $\left(\mathrm{mm}^{2}\right)$ & 17,0 & & 1,0 & & & & & & 201,3 \\
\hline
\end{tabular}

+ TL 50 - Tempo necessário para que haja $50 \%$ de mortalidade.

++- Dados fornecidos pelo Instituto Nacional de Metereologia.

+++- Não atingiu $50 \%$ de mortalidade em 30 dias.

ND - Nào há dados.

Tabela 3-Dados de $\mathrm{TL}_{50}{ }^{+}$e temperatura média, umidade relativa do are pluviosidade no Distrito Federal ++ . Material borrifado em 15/10/1981.

\begin{tabular}{|c|c|c|c|c|c|c|}
\hline \multirow{2}{*}{$\begin{array}{l}\text { Material e dados } \\
\text { Meteorológicos }\end{array}$} & \multicolumn{6}{|c|}{ Dias após a borrifação } \\
\hline & 6 & 143 & 240 & 340 & 418 & 477 \\
\hline Capim de arroz & 1 & 2 & 23,5 & 22,5 & 13 & 5 \\
\hline Acrocomia sclerocarpa & 1 & 4 & 20,5 & 17 & 13 & 8,5 \\
\hline Tijolo não cozido & 1 & 1,5 & 4 & 12 & 3 & 2 \\
\hline Adobe & 1 & 1 & 11,5 & 14,5 & 3,5 & 2 \\
\hline Telha & 1 & 1,5 & 8,5 & 15 & 6 & ND \\
\hline Madeira & 1 & 4 & +++ & +++ & 14 & 3,5 \\
\hline Mauritia vinifera & 1 & 3,5 & 12,5 & 15 & 8,5 & 4 \\
\hline Reboco com cal & 1 & 3 & 22 & 23 & 13 & 7,5 \\
\hline Reboco sem cal & 1 & 1,5 & 14 & 19 & 6 & 3,5 \\
\hline Mortalidade controle & +++ & +++ & +++ & +++ & +++ & +++ \\
\hline \multicolumn{7}{|l|}{ Temperatura média } \\
\hline$\left({ }^{\circ} \mathrm{C}\right)$ & 20,5 & 21,1 & 19.4 & NDD & 20.9 & 21 \\
\hline \multicolumn{7}{|l|}{ Umidade relativa } \\
\hline$(\%)$ & 58 & 81 & 62 & ND & ND & 84 \\
\hline Pluviosidade $\left(\mathrm{mm}^{3}\right)$ & 437,5 & 233,4 & 0 & 80,4 & 160,2 & 207,3 \\
\hline
\end{tabular}

$+\mathrm{TL}_{50}$-Tempo necessário para que haja $50 \%$ de mortalidade.

++ - Dados fornecidos pelo Instituto Nacional de Metereologia.

+++- Não atingiu $50 \%$ de mortalidade em 30 dias.

ND - Não há dados. 


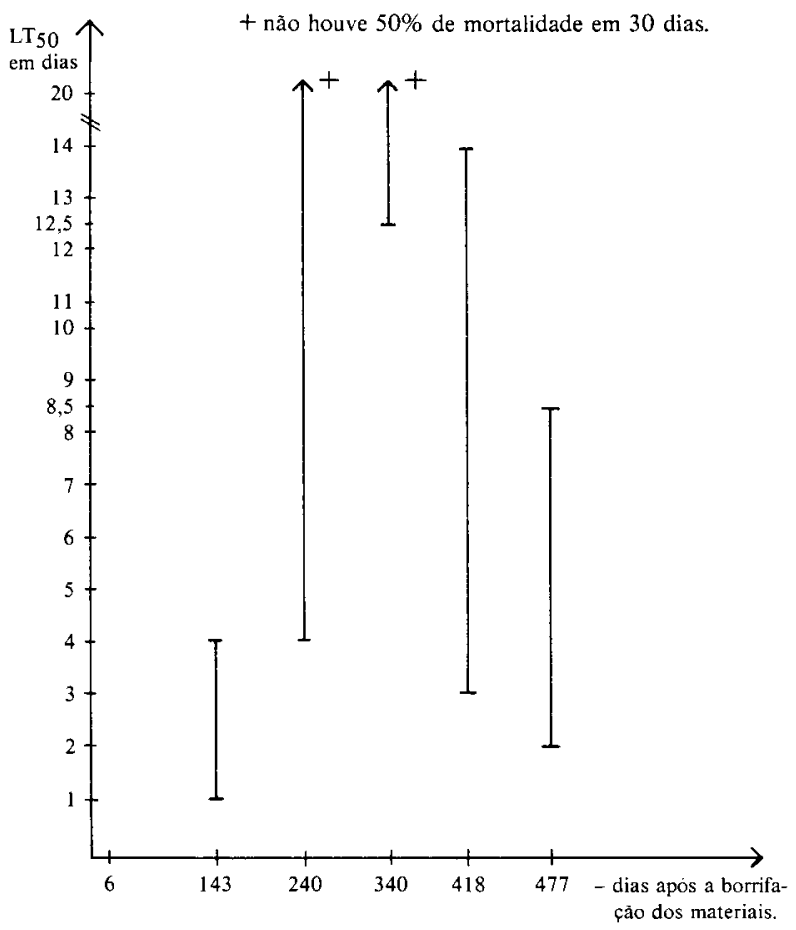

FIG. 1 - Gráfico LT $_{50}$ em dias e tempo apos a borrifação com BHC dos materiais em 15.10 .81

\section{DISCUSSÃO}

A aplicação dc BHC em casas é um procedimento de rotina no combate aos barbeiros, utilizado desde a descoberta de sua ação residual contra triatomineos 11215 . Os estudos realizados mostram que, sob nossas condições de teste, a atividade residual do BHC pode ser detectada até 627 dias após a borrifação. $O$ estudo inicial no campo (Tabela 1) mostrou que após 7 meses o TL50 nas superficies testadas era atingido, dentro de uma semana, com exceção da casa 7. Sabemos que as condiçōes de teste são artificiais, já que, na natureza, o contato do barbeiro com a superficie borrifada é apenas transitorio, quando ele anda sobre ela à procura de alimento. Mas, para o nosso objetivo - estudar o efeito residual do $\mathrm{BHC}$ a longo prazo - necessitávamos do sistema teste o mais sensivel possivel. Afirma-se que o BHC perde a sua toxicidade em 3 meses, razão pela qual são recomendadas borrifações repetidas pelo Ministério da Saúde. Dias ${ }^{1}$ mostrou mortalidade uniforme em ninfas de primeiro estagio de Triatoma infestans e Panstrongylys megistus, cinco meses após a aplicação de BHC a papéis de filtro.

Nas Tabelas 2 e 3 podem ser observadas marcantes diferenças entre os vários tipos de materiais de construção no tocante à sua capacidade de expressar a atividade do inseticida na superficie. Uma semana após a borrifação foi observada mortalidade uniforme em todas as superficies. Observaçōes mais tardias mostraram, contudo, uma grande variação na capacidade de induzir mortalidade de um mesmo material e entre materiais diferentes. Esta variação, que também esteve presente no experimento de campo, está provavelmente relacionada à umidade ambiental, já que há uma correlação entre a pluviosidade do mès e a atividade do $\mathrm{BHC}$ nos materiais de teste. Este dado foi observado previamente para o $\mathrm{DDT}^{5}$, sendo que as relaçōes umidade-temperatura, expressas como umidade relativa do ar, exercem uma poderosa influencia na atividade do inseticida 16 . Nocerino ${ }^{13}$ mostrou, recentemente, a reativação do Dieldrin após a umidificação de blocos de barro aspergidos com inseticida 130 dias antes. O gama BHC é absorvido pelos blocos de barro e quase toda a dose de cristais de 10 a 20 microns pode ser eventualmente recuperada do interior dos blocos 7 . $\mathrm{O}$ aumento da unidade provoca o movimento destes cristais de volta à superficie. Uma correlação positiva pode ser observada entre a atividade residual do DDT na superfície de barro e o conteúdo relativo de quartzo da fração coloidal do adobe. Um aumento no conteúdo de kaolinite, por outro lado, causou uma diminuição na atividade residual do DDT. A velocidade de absorção do BHC na superficie depende do tipo de barro 314 .

Obviamente, a atividade residual dos inseticidas está relacionada a uma serie de fatores ambientais complexos, assim como, à natureza quimica do inseticida.

Pretendemos aprofundar nossos estudos a fim de reproduzir as condições de campo, nas quais o triatomineo entra em contato apenas transitório com o inseticida, para verificar se a prolongada atividade residual do $\mathrm{BHC}$ demostrada neste trabalho tem algum valor pratico no combate a triatomíneos.

\section{$S U M M A R Y$}

In Mambai (Goiás) the population use various types of building materials. Since a variation in the persistence of BHC activity has been noted in different materials, laboratory experiments were designed to investigate this aspect. The experiments employed a test kit of ten first instar Dipetalogaster maximus and verified the time necessary to achieve $50 \%$ mortality ( $L T_{50}$ ) after continuous contact with 
Penna R, Bosworth A, Brasil IA, Marsden PD. Persistencia da atividade residual do BHC na superficie de diferentes materiais de construção. Revista da Sociedade Brasileira de Medicina Tropical 17; 95-99, Abr-Jun, 1984

the sprayed surface. The tests were always done in duplicate with adequate controls. Thirty-two surface from ten types of materials were tested at intervals up to 627 days after spraying.

The results obtained can be summarized as follows: 1) the residual activity of $B H C$ could be detected in our test system up to the final observation, 627 days after the test; 2) this activity varied greatly among the different materials 88 days after spraying. This variation was also related to variations in the atmospheric relative humidity; and 3) the capacity of the materials to retain the residual activity of $B H C$ in this system was the following in the order most efficient to least: adobe mud and unfired brick, tile, palm fronds and plaster without lime, straw and plaster with lime, fired brick and wood.

Key words: Insecticide. Residual activity. Triatomines. BHC.

\section{AGRADECIMENTOS}

Agradecemos ao senhor Domingos das Virgens a insubstituivel assistência no trabalho de campo; ao professor Aluizio Prata pelo fornecimento das ninfas de 1 . estágio de $D$. maximus, indispensáveis para a realização dos experimentos; ao Instituto Nacional de Meteorologia pelo fornecimento dos dados meteorológicos; e ao Conselho Nacional de Desenvolvimento Científico e Tecnológico $(\mathrm{CNPq})$ pela ajuda financeira.

\section{REFERÊNCIAS BIBLIOGRÁFICAS}

1. Dias JCP. Suscetibilidade de larvas e ovos de triatomineos à ação do BHC. Revista Brasileira de Malariologia e Doenças Tropicais 17: 37-47, 1965.

2. Dias E, Pellegrino J. Alguns ensaios com o gammexane no combate aos transmissores da doença de Chagas. Brasil Médico 62: 185-190, 1948.

3. Fromm AS, Gandini P. Absorção do isomero gama do BHC por diferentes tipos de barro. Avaliação biológica com Triatoma infestans. Revista Brasileira de Malariologia e Doenças Tropicais 23: 111-134, 1971.

4. Garron A, Bermudez H, Muynk A. Pruebas biológicas de pared y ensayo de campo con propoxur y BHC. Boletin Informativo de CENETROP V: 37-46, 1979.

5. Hadaway AB, Barlow F. The influence of temperature and humidity upon the action of insecticides during the post-treatment period. Annals of Tropical Medicine and Parasitology 51: 187-193, 1957.
6. Hadaway AB, Barlow F. The residual action of two organophosphorus compounds and a carbamate on dried muds. Bulletin of the World Health Organization 28: 69$76,1963$.

7. Hadaway AB, Barlow F. Studies on aqueous suspensions of insecticides. Part III: factors affecting the persistence of some synthetic insecticides. Bulletin of Entomological Research 43: 291-311, 1952.

8. Martinez A, Cichero JA, Alania IR, Gonzalez FF. Control of Triatoma infestans (Klug) with malathion concentrate. Journal of Medical Entomology 11: 653 $657,1973$.

9. Marsden PD, Alvarenga NJ, Cuba CC, Shelley AJ, Costa $\mathrm{CH}$, Boreham PFL. Studies of the domestic ecology of Triatoma infestans by means of house demolition. Revista do Instituto de Medicina Tropical de São Paulo 21: 13-25, 1975.

10. Marsden PD, Virgens D, Magalhães I, Tavares-Neto J, Ferreira R, Costa CHN, Macedo V, Prata A. Ecologia doméstica do Triatoma infestans em Mambaí, GoiásBrasil. Revista do Instituto de Medicina Tropical de São Paulo 24: 364-375, 1982.

11. Marsden PD, Virgens D, Castro CN, Brasil IP, Ferreira R, Silveira AC, Matos CAS, Macedo V, Prata A. The control of Chagas' disease in Mambai, Goiás-Brasil (1980-1981). Revista da Sociedade Brasileira de Medicina Tropical 16: 189-195, 1983.

12. Ministério da Saúde. Manual de normas técnicas da campanha de controle da doença de Chagas. Centro de Documentação do Ministério da Saúde, Brasília, 1980.

13. Nocerino F. Afloramiento de cristales de insecticidas con el humedecimiento en las superficies rociadas. Boletin de la Direccion de Malariologia y Saniamiento Ambiental 21: 54-58, 1981.

14. Paulini E, Murta CC, Pereira JP. Novos estudos sobre a desativação do DDT em superficies de barro. Revista Brasileira de Malariologia e Doenças Tropicais 18: 99-104, 1966.

15. Romaña C, Abalos JR. Accion del gammexane sobre los triatomideos. Controle domiciliario. Anais del Instituto de Medicina Regional del Tucuman 2: 95-106, 1948.

16. Romeiro L, Aguiar H. Ação da temperatura sobre alguns inseticidas. Revista Brasileira de Malariologia e Doencas Tropicais 6: 131-143, 1954.

17. Sherlock IA, Piesman J, Guitton N. Action of bendiocarb against triatomine (Hemiptera, Reduviidae) vectors of Chagas' disease. Journal of Medical Entomology 20: 440-445, 1983. 